\title{
Estado actual de la asistencia técnica y su incidencia en el desempeño agrícola en el departamento de bolívar, Colombia
}

\section{Actual condition of technical assistance and incidence on agriculture performance in bolivar, Colombia}

\author{
Francisco Javier Maza - Avila1, Jeraldin Fuentes - Maturana ${ }^{2}$, \\ Jessica Paola Franco - Santamaría ${ }^{3}$ \\ ${ }^{1}$ Grupo de investigación en estudios para el Desarrollo Regional -GIDER-, Universidad de Cartagena, Colombia, \\ ORCID: https://orcid.org/0000-0002-3936-8246, E-mail: fmazaa@unicartagena.edu.co \\ ${ }^{2}$ Administradora Industrial, Universidad de Cartagena, Colombia, \\ ORCID: https://orcid.org/0000-0002-8447-9535, E-mail: jfuentesmaturana16@gmail.com \\ ${ }^{3}$ Administradora Industrial, Universidad de Cartagena, Colombia, \\ ORCID: https://orcid.org/0000-0001-9495-0944, E-mail: jesii_2796@ hotmail.com
}

Cómo citar: Maza - Avila, F. J., Fuentes -Maturana, J., \& Franco - Santamaría, J. P. (2019). Estado actual de la asistencia técnica y su incidencia en el desempeño agrícola en el departamento de Bolívar, Colombia. Revista Científica Profundidad Construyendo Futuro, 10(10), 31-41. https://doi.org/10.22463/24221783.2575

Recibido: 03 de Octubre de 2018/ Aprobado: 15 de Diciembre de 2018

\section{Resumen}

Debido a la importancia del sector agrícola y las necesidades de incremento de su productividad y competitividad, el objetivo de este artículo consiste en caracterizar el estado actual de la asistencia técnica entre productores agrícolas de departamento de Bolívar, así como su incidencia en el rendimiento final de sus cultivos. Para ello, se ha acudido a las estadísticas del III Censo Nacional Agropecuario del año 2014, así como a la base de datos del Sistema de Información Agrícola -AGRONET- del Ministerio de Agricultura y Desarrollo Rural para el periodo entre el año 2010 - 2018. Los resultados arrojan que son pocos los productores agrícolas del departamento de Bolívar que reciben un servicio de asistencia técnica, respondiendo a la tendencia a nivel nacional. Por su parte, no se hallaron diferencias significativas del desempeño agrícola entre los productores agrícolas que reciben el servicio de asistencia técnica respecto a aquellos que no lo reciben.

Palabra clave: Asistencia técnica, Agricultura colombiana, Desempeño agrícola, Desarrollo endógeno.

\section{Abstract}

The objective of this article is to characterize the current state of technical assistance among agricultural producers in the department of Bolivar, as well as its impact on the final yield of their crops. For this purpose, the statistics of the III National Agricultural Census have been used, as well as the database of the Agricultural Information System AGRONET- of the Ministry of Agriculture and Rural Development. The results show that few agricultural producers in the department of Bolívar receive technical assistance service, responding to the trend at the national level. On the other hand, no significant differences in agricultural performance were found between agricultural producers who receive the technical assistance service compared to those who do not.

Keyword: Technical Assistance, Colombian Agriculture, Agricultural Performance, Endogenous Development.

*Autor para correspondencia.

Correo electrónico: fmazaa@unicartagena.edu.co

La revisión por pares es responsabilidad de la Universidad Francisco de Paula Santander Ocaña

Artículo bajo licencia CC BY-NC (https://creativecommons.org/licenses/by-nc/4.0/) 


\section{Introducción}

La agricultura es una de las actividades económicas que más aporta al desarrollo, la soberanía, la seguridad alimentaria, así como a la reducción de la pobreza, mediante la generación de empleo en el sector rural de un país (Valdés et al., 2008), de allí la importancia de mejoras de tecnificación en la práctica agrícola (Perfetti et al., 2013). Pese a ello, el sector agrícola de Latinoamérica en los últimos años ha tenido un comportamiento de decrecimiento, y por ello se debe seguir promoviendo las inversiones en investigación, desarrollo, educación rural $\mathrm{y}$ servicios de asistencia técnica e infraestructura para aumentar su productividad (Cepal et al., 2017). Para la región de América Latina, los acuerdos con los Estados Unidos se convierten en un gran reto, dada la incertidumbre que genera los intercambios diarios; no obstante, estos países dependen del crecimiento de la demanda de materias primas, o comodities. A pesar que han aumentado su participación en el mercado mundial, los países se ven presionados a implementar medidas que impulsen aún más el comercio para cumplir con la demanda del mercado (Cepal et al., 2017).

Históricamente, el sector agrícola ha sido una actividad prioritaria en la economía colombiana, dada la generación de empleo y su aporte al desarrollo rural; no obstante, el sector presenta deficiencias en infraestructura, productividad y competitividad en los mercados internacionales (Lozano y Restrepo, 2015). Debido a esto, y con la finalidad de mitigar el problema e impulsar el movimiento del sector, el gobierno de Colombia ha implementado un programa que permite a los agricultores mejorar las técnicas aplicadas en su sistema de producción, orientado a sus capacidades, el cual se desarrolla bajo principios que garanticen el desarrollo productivo (Procasur et al., 2016). Este servicio de asistencia técnica busca hacer a las Unidades Productivas Agrícolas -UPA'smás competitivas (Leibovich et al., 2010). Por ello, se espera que las UPA's que reciben el servicio de asistencia técnica sean más productivas que aquellas que no lo reciben.

Bolívar, departamento clave que conforma parte de la región Caribe colombiana, se encuentra clasificado como uno de los departamentos de clase mundial, al contar con estructuras productivas diversificadas con capacidad competir en mercados internacionales (Gobernación de Bolívar, 2010). Asimismo, con el fin de contribuir a la mejora de sus sectores estratégicos, el departamento ha trabajado en su plan de competitividad, proponiendo metas a mediano y largo plazo para el fortalecimiento de todos sus sectores, entre ellos el agrícola (Gobernación de Bolívar, 2010). Entre las acciones planificadas se encuentra el fortalecimiento de la asistencia técnica en el sector agrícola, con la finalidad de incrementar su competitividad nacional e internacional.

Ante la situación planteada, surgen varios interrogantes para este artículo ¿Cuál es el estado actual de la asistencia técnica a nivel del departamento de Bolívar? ¿Tiene incidencia el servicio de asistencia técnica del departamento de Bolívar en su desempeño agrícola? En tal sentido, este artículo tiene como objetivo central caracterizar el estado actual de la asistencia técnica y su incidencia en el desempeño agrícola en el departamento de Bolívar. Para responder a lo anterior, este artículo se ha dividido en las siguientes secciones: después de esta introducción, se describe la situación actual del sector agrícola 
colombiano y de Bolívar, seguido el marco teórico y conceptual sobre la relación entre la asistencia técnica agrícola y la competitividad, luego, en términos de asistencia técnica. El siguiente apartado corresponde a la metodología empleada en este artículo, le siguen los resultados obtenidos en materia de asistencia técnica y su relación con la productividad a nivel del departamento. Al final, se exponen las conclusiones y las referencias bibliográficas.

\subsection{Situación actual del sector agrícola colombiano}

Colombia es un país rico en recursos naturales, tanto renovables como no renovables, entre los más importantes está el oro, el petróleo, flora y fauna, Asimismo, cuenta con una gran variedad de cultivos y productos forestales, diversidad de climas y bosque tropicales (OCDE, 2014). Quien está a cargo del sector agrícola a nivel nacional es el Ministerio De Agricultura y Desarrollo Rural, el cual para cumplir con las exigencias del mercado mundial y el crecimiento del sector, su prioridad es invertir en innovación y conocimiento (Maza Avila, 2016; Procasur et al., 2016). Es preciso destacar que el empleo en el sector agropecuario es un factor de suma importancia, para así mitigar la pobreza rural (Corredor, 2017).

Sin embargo, el sector agrícola Colombiano está sujeto a varios problemas, reflejados en la productividad del mismo, entre esos está la infraestructura vial y los accesos de vías terciarias, las técnicas agrícola empleadas por los productores, la calidad de vida y el uso del suelo (Castaño Giraldo y Cardona Gómez, 2014).
Según Villanueva Mejía (2018), la participación de la producción anual del sector agropecuario en el PIB nacional para el año 2016 contribuyó a la economía en un 9\% de la producción total. Colombia cuenta con 40,3 millones de hectáreas para uso agropecuario, de las cuales 8,5 millones son para uso agrícola $(19,7 \%)$ y de estas hectáreas el $83,9 \%$ corresponde a cultivos agrícolas (Departamento Administrativo Nacional de Estadística) [DANE, 2016]. De igual manera, se destaca que en el país se siembran aproximadamente 223 productos, pero una baja porción de ellos -sólo 12- representan el $86 \%$ del total del área sembrada.

Tabla 1. Participación (\%) del área sembrada por productos a nivel nacional $2010-2018$

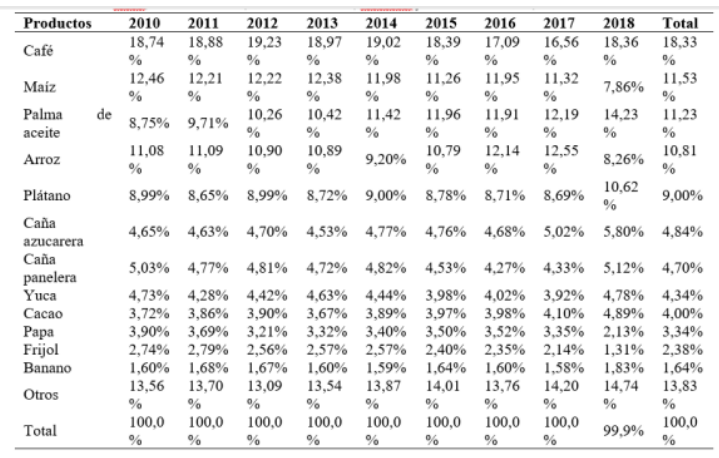

Fuente: Elaboración propia, a partir del sistema de información agrícola AGRONET.

Entre los productos más representativos están el café, el maíz, la palma de aceite, el arroz y el plátano. En la Tabla 1 se muestra que el café en los últimos ocho años ha mantenido su participación por encima de los $18,0 \%$ puntos porcentuales, siendo el producto más representativo en Colombia. Por su parte, el maíz para el año 2018 disminuyó su participación con un 7,86\% en el área sembrada. 
Cabe resaltar que, en los últimos ocho años, la participación de la palma de aceite respecto a las hectáreas sembradas ha venido creciendo. Tal como lo sostienen Herrera y Cumplido (2015) en su investigación sobre las implicaciones del cultivo de palma de aceite en la estructura productiva agrícola y la seguridad alimentaria de María La Baja, la palma ha sido el producto con el mayor crecimiento en los últimos años. Además, los productos con mayor participación a nivel nacional en el número de hectáreas sembradas son de ciclo permanente con una participación del 59\%, continuo a eso los productos transitorios con un 35\% y anual 6\% (Figura 1).

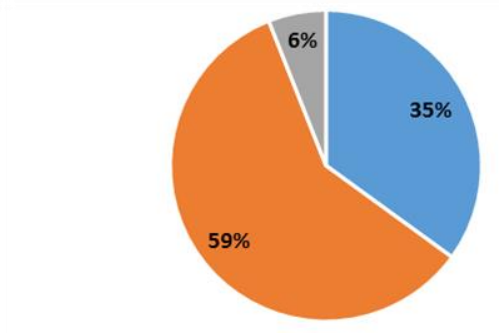

" Cultivos transitorios $=$ Cultivos anuales $=$ Cultivos permanentes

Figura 1. Participación nacional del ciclo de cultivo 2010 - 2018. Fuente: elaboración propia, a partir del sistema de información agrícola AGRONET.

En términos de asistencia técnica, sólo el $16,5 \%$ declaró haber recibido el servicio, mientras que el 83,5\% sostiene que no. Así mismo, el III Censo Nacional Agropecuario del año 2014 arroja que los tipos de asistencia técnica que más se ofrece a nivel nacional son buenas prácticas agrícolas, comercialización, crédito y financiamiento, tal como lo muestra la Figura 2.

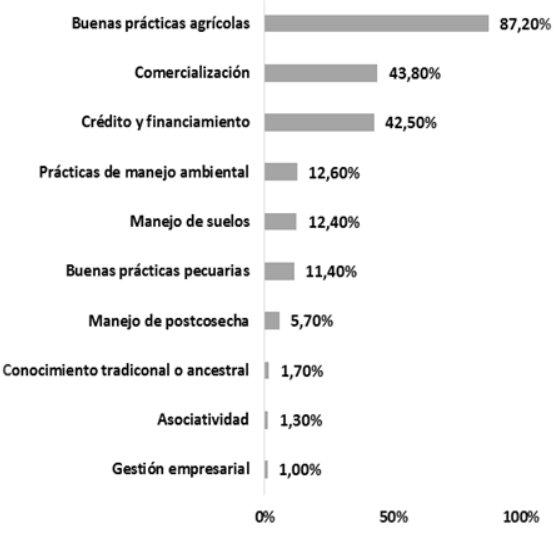

Figura 2. Distribución (\%) de UPA en el área rural dispersa censada con asistencia técnica recibida, según tipo de asistencia. Fuente: elaboración propia, a partir de las estadísticas del Tercer Censo Nacional Agropecuario.

\section{Marco teórico}

El concepto de desarrollo se define como un conjunto de aptitudes o capacidades intrínsecas de cada región, que deben ser descubiertas para su mejor aprovechamiento (Berton, 2009). Para el caso del desarrollo rural, (Alfaro, 2013) lo concibe como un proceso donde la transformación no solo es productiva, sino también institucional y social, bases importantes para la búsqueda del bienestar de la población en cuestión. En el transcurso de los años, la productividad agrícola ha jugado un papel importante a favor del desarrollo, la economía, y de la lucha contra la pobreza (JIA \& IICA, 2015). Es preciso mencionar que la productividad es la relación entre los recursos empleados dentro del proceso productivo y los recursos que se obtienen (Carro \& González, 2012).

La productividad agrícola para una región puede concebirse, por tanto, como la relación entre los resultados que se obtienen en la agricultura respecto a los factores totales de la producción: tierra, agua, capital, entre otros 
insumos de material (JIA \& IICA, 2015). Sin embargo, la agricultura no consiste únicamente en la obtención de productos vegetales, sino que también incluye el cuidado y la conservación de la tierra, considerándola el mayor recurso para la productividad y que le permitirá al comprador disfrutar de un producto saludable $\mathrm{y}$ de calidad (ICA, 2016).

La asistencia técnica se constituye en uno de los factores que puede apoyar el incremento de la productividad agrícola, ya que su objetivo está encaminado a mejorar el rendimiento de la producción de alimentos básicos o actividades que tienen como unidad productora la tierra, a través de asesorías relacionadas con procesos de producción, industrialización, producción y comercialización (Cuevas Reyes, Baca del Moral, Cervantes Escoto, \& Aguilar Ávila, 2018). La asistencia técnica es uno de los principales servicios requeridos por los agricultores, ya que el acceso a este contribuye en el mejoramiento a nivel competitivo de las exportaciones agrícolas (Landini, 2016). Por lo tanto, y con el fin de mejorar la competitividad del sector, es necesario contar con políticas y programas de asistencia técnica que incorporen nuevas técnicas, y métodos que incrementen los rendimientos agrícolas (Castaño Giraldo \& Cardona Gómez, 2014), de modo que beneficie a quienes dedican su labor a la explotación agrícola.

En Colombia, la asistencia técnica adquiere relevancia con el Decreto No 2379 de 1991, que en su artículo $\mathrm{N}^{\circ} 3$ la define como un servicio de transferencia tecnológica la cual se brinda a los pequeños productores mediante asesoría, consultoría, capacitación y aplicación de métodos que contribuyan con el mejoramiento de los sistemas de producción de las explotaciones rurales, así como mejorar los niveles de ingresos y la capacidad productiva de la población campesina (Ministerio de Agricultura y Desarrollo Rural, 1991). Posteriormente, la Ley 607 del 2000 , lo estipula como un servicio directo y obligatorio, además, señala la necesidad de racionalizar y coordinar actividades que le correspondan, asegurando una ampliación, cobertura, calidad y pertinencia del servicio de orientación y acompañamiento (Ministerio de Agricultura y Desarrollo Rural, 2000). Existen varias entidades prestadoras del servicio de extensión y asistencia técnica como son las Unidades Municipales De Asistencia Técnica Agropecuaria UMATA's-, los Centros Provisionales De Gestión Agropecuaria -CPGA-, empresas privadas, gremios agropecuarios y demás entidades cuya razón social sea la prestación del servicio (Ministerio de Agricultura y Desarrollo Rural, 2000).

Para el año 2016 se logra firmar el acuerdo final para la terminación del conflicto y la construcción de una paz estable y duradera con las Fuerzas Armadas Revolucionarias de Colombia FARC. Con referencia a lo anterior, el primer punto del acuerdo fue la Reforma rural integral - Hacia un nuevo campo colombiano, en este se determina la creación del Plan nacional de fomento a la economía solidaria y cooperativa rural, el cual tendrá criterios como acompañamiento, apoyo técnico y financiero, fortalecer las capacidades productivas y los instrumentos para el desarrollo rural como lo es la asistencia técnica, formación y capacitación. En ese sentido, el mismo acuerdo el gobierno nacional se compromete a la creación de un plan nacional de asistencia técnica integral (Presidencia de la República \& FARC-EP, 2016). 


\section{Metodología}

La investigación de este artículo es de tipo descriptivo y correlacional, y para su desarrollo se tomó como población a los productores agrícolas de los 46 municipios del departamento de Bolívar. Para ello, se acudió a la base de datos anonimizados del III Censo Nacional Agropecuario, elaborado por el Departamento Administrativo Nacional de Estadística -DANE- en el año 2014, cuyos datos fueron publicados en el año 2016. También se acudió a la base de datos estadística del Sistema de Información Agrícola - AGRONET-, para el periodo entre el año 2010 - 2018, con el fin de caracterizar el sector agrícola en función de las hectáreas sembradas, hectáreas cosechadas, niveles de producción, principales productos sembrados en la región y el ciclo del cultivo.

Los datos recolectados del III Censo Nacional Agropecuario del año 2014 para el departamento de Bolívar inicialmente fueron de 54.346 datos, los cuales pasaron por un proceso de depuración, con la finalidad de eliminar datos atípicos y encuestas que no fueron respondidas; de esta depuración se eliminaron 31.182 datos, para dar con un total de 23.164 datos finales, los cuales se utilizaron para caracterizar el estado actual de la asistencia técnica y su incidencia en el desempeño agrícola en el departamento de Bolívar. Las variables que fueron consideradas para el análisis fueron las siguientes: Producción agrícola, expresada en toneladas/hectáreas, Buenas prácticas agrícolas $\left(\mathrm{X}_{1}\right)$, Prácticas de manejo ambiental $\left(\mathrm{X}_{2}\right)$, Manejo de suelo $\left(\mathrm{X}_{3}\right)$, Manejo de poscosecha $\left(\mathrm{X}_{4}\right)$, Comercialización $\left(\mathrm{X}_{5}\right)$, Asociatividad $\left(\mathrm{X}_{6}\right)$, Crédito y financiamiento $\left(\mathrm{X}_{7}\right)$, Gestión empresarial $\left(\mathrm{X}_{8}\right)$. En la Tabla 2 se muestran las variables utilizadas para este artículo.
Tabla 2. Definición de las variables

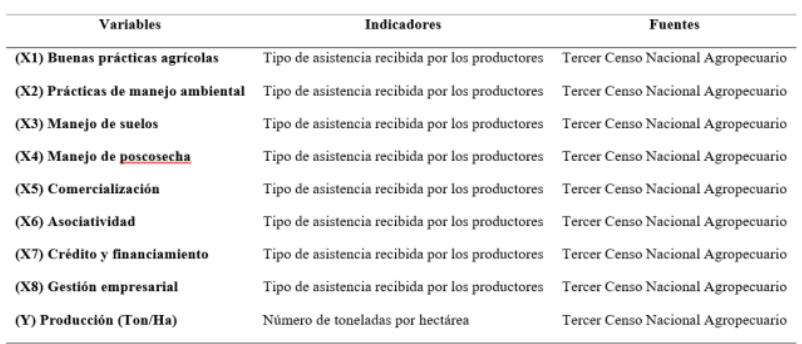

Fuente: Elaboración propia, a partir del III Censo Nacional Agropecuario del año 2014.

\section{Resultados}

Este artículo tuvo como objetivo la caracterización del estado actual de la asistencia técnica del departamento de Bolívar y su incidencia en el desempeño agrícola de las Unidades Productivas Agrícolas -UPA's-. En Bolívar, en relación con el área sembrada, los resultados arrojan que solo nueve productos representan el 93,74\% del total, entre estos productos los más representativos son el Maíz, la Yuca, y la Palma de aceite (Tabla 3), siendo este último cultivo el de mayor crecimiento durante los últimos años. En la actualidad, la mayoría de las hectáreas cosechadas de palma de toda la región se concentran en el municipio de María La Baja, representando así el 35,08\% de su estructura productiva.

Tabla 3. Participación (\%) del área sembrada por productos en el departamento de Bolívar 2010 - 2018

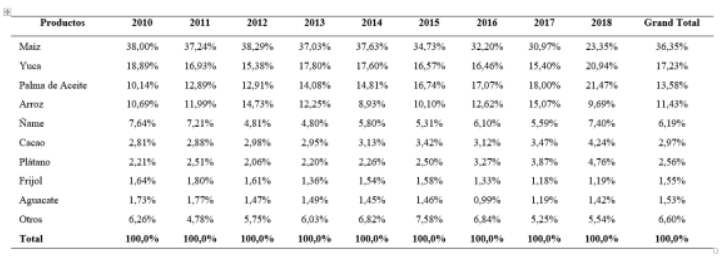

Fuente: Elaboración propia, a partir de las estadísticas del Tercer Censo Nacional Agropecuario (2014). 
Se espera que el crecimiento de este cultivo continúe, en detrimento del resto de cultivos, debido al marco regulatorio nacional, entre ellos la Ley 939 de 2004 modificatoria de la Ley 818 de 2003- que brinda beneficios de exención de la renta para los ingresos derivados del aprovechamiento de cultivos de tardío rendimiento -que incluye a los cultivos de cacao, caucho, palma de aceite, cítricos, y frutales- por 10 años siguientes a partir del inicio de la producción (Herrera Seba \& Cumplido Hernández, 2015).

Por otra parte, se analizó la participación del ciclo de cultivo en el área cosechada y, en promedio, en Bolívar la realidad de los productos de ciclo de cultivo permanente como la Palma de aceite se acentúa mucho más, teniendo en promedio un $58,69 \%$ de participación en el área cosechada, mientras que muy por debajo se encuentra los productos con ciclo anual $(5,60 \%)$ y con un $35,71 \%$ los productos de ciclo transitorio (Tabla 4).

Los resultados anteriores ponen de manifiesto la riqueza del suelo del departamento, en general, así como también la preocupación por el fuerte ascenso en el crecimiento de la palma de aceite, lo que requerirá tomar medidas que contribuyan al desarrollo sostenible de la región, sin poner en riesgo su seguridad alimentaria.

Tabla 4. Tabla 3 Participación (\%) del área sembrada por productos en el departamento

\begin{tabular}{|c|c|}
\hline Ciclo cultivo & Promedio \\
\hline Permanente & $58,69 \%$ \\
\hline Transitorio & $35,71 \%$ \\
\hline Anual & $5,60 \%$ \\
\hline Total & $100 \%$ \\
\hline
\end{tabular}

Fuente: Elaboración propia, a partir del sistema de información agrícola AGRONET (2010 - 2018).
En cuanto al productor Bolivarense, a nivel nacional los resultados muestran que son muy pocos los productores que señalan la agricultura como una actividad rentable. Por ello, y para generar recursos adicionales, necesarios para cubrir los gastos del hogar, el $61,11 \%$ de los productores realizan actividades paralelas, tales como la albañilería, la cría de animales o actividades comerciales, caracterizadas por ser esporádicas e informales, conocidas en Colombia como "rebusque" (Maza Avila, 2016).

Por otra parte, si bien existe una formalidad en la tenencia de las UPA's, al observar la naturaleza jurídica para el ejercicio de su actividad productiva, son evidentes los niveles de informalidad empresarial. A nivel de Bolívar, el 99,8\% de los productores residentes en el área rural son personas naturales, concentrándose el $64,9 \%$ en UPA menores a 5 hectáreas.

A nivel regional, la producción agrícola del municipio se caracteriza por sus bajos niveles de tecnificación y por el empleo intensivo de mano de obra en todas las fases de producción agrícola. Es usual que la mano de obra esté conformada por miembros de la familia -padres, hermanos, sobrinos, entre otros- y sólo se acuda a mano de obra adicional cuando la actividad a realizar así lo requiera, algo que ocurre con mayor frecuencia en la fase de preparación del terreno. Los anteriores resultados pueden tener una incidencia en el acompañamiento y asistencia técnica. De hecho, en términos de asistencia la situación no es diferente respecto a la nacional, dado que solo el 10,9\% de los productores censados declaran haber recibido asistencia técnica, Es decir que, del total de encuestados en el departamento de Bolívar 49.241, solo 5.383 productores declararon haber recibido asistencia técnica (Tabla 5). 
Estado actual de la asistencia técnica y su incidencia en el desempeño agrícola en el departamento de bolívar

Tabla 5. Porcentaje total área censada en el departamento de Bolívar con asistencia técnica recibida

\begin{tabular}{cccc}
\hline Departamento & Total & Sí recibió & $\begin{array}{c}\text { No } \\
\text { recibió }\end{array}$ \\
\hline Total Bolívar & 49.241 & 5.383 & 43.858 \\
& $100.0 \%$ & $10.9 \%$ & $89.1 \%$
\end{tabular}

Fuente: Elaboración propia, a partir de las estadísticas del Tercer Censo Nacional Agropecuario (2014)

De acuerdo al III Censo Nacional Agropecuario, el tipo de asistencia técnica, para aquellos que sí la recibieron, se concentró, sobre todo, en buenas prácticas agrícolas y asistencia en crédito y financiamiento, siendo casi nulas las orientadas al manejo de la post-cosecha, la comercialización, la asociatividad, la gestión empresarial, aspectos que resultan claves para apalancar el proceso productivo y, además, lograr mejores márgenes de ganancia (Tabla $6)$.

Tabla 6. Total de UPA's en el área rural dispersa censada con asistencia técnica recibida en el Departamento de Bolívar, según tipo de asistencia

\begin{tabular}{lc}
\hline Tipo de asistencia técnica & No. \\
\hline Buenas prácticas agrícolas & 4.769 \\
Crédito y financiamiento & 2.504 \\
Manejo de suelos & 444 \\
Prácticas de manejo ambiental & 275 \\
Comercialización & 255 \\
Manejo de poscosecha & 218 \\
Asociatividad & 65 \\
Gestión empresarial & 42 \\
\hline
\end{tabular}

Fuente: Elaboración propia, a partir de las estadísticas del Tercer Censo Nacional Agropecuario (2014)

Luego de realizar un análisis del promedio del rendimiento medido en Ton/Ha del total de UPA's encuestadas en el departamento de Bolívar, se encontró que los productores que no perciben el servicio de asistencia técnica son 0,54 puntos más productivas que aquellos que si declararon haber recibido el servicio de asistencia técnica (Tabla 7).

Tabla 7. Promedio de rendimiento total de las UPA's respecto a la percepción del servicio de asistencia técnica

\begin{tabular}{cc}
\hline Recibe asistencia técnica & $\begin{array}{c}\text { Promedio Rendimiento } \\
\text { (Ton/ Ha) }\end{array}$ \\
\hline $\mathrm{Si}$ & 2,83 \\
$\mathrm{No}$ & 3,37 \\
Total & 3,27 \\
\hline
\end{tabular}

Fuente: Elaboración propia, a partir de las estadísticas del Tercer Censo Nacional Agropecuario (2014)

De hecho, los resultados del cálculo del Coeficiente de Determinación (R2) para cada una de las variables analizadas, evidencia que las variables explicativas tienen poca incidencia con la variable dependiente, ya que los valores del R2 están más cerca a cero y, por lo tanto, su significancia es relativamente nula y el modelo no puede ser explicado. (Tabla 8).

Tabla 9. Estadístico de regresión - $R 2$ coeficiente de determinación

\begin{tabular}{cc}
\hline Variables & R2 \\
\hline X1 & 0.002444 \\
X2 & 0.002376 \\
X3 & 0.002376 \\
X4 & $4.33 \mathrm{E}-05$ \\
X5 & $1.02 \mathrm{E}-05$ \\
X6 & 0.002723 \\
X7 & 0.001223 \\
X8 & 0.001136 \\
\hline
\end{tabular}

Fuente: Elaboración propia.

\section{Discusiones}

Este trabajo arroja que la asistencia técnica recibida incide en el desempeño de UPA's de manera mínima. Dichos resultados están en 
sintonía con las conclusiones del trabajo realizado por Maza, Batista \& Rodríguez (2018) para las UPA's de la región Caribe colombiana. Vale señalar, sin embargo, que tanto el trabajo de Cuevas et al. (2018) realizado en unidades de producción en México, como el de Leibovich et al. (2010) en su investigación sobre el proceso de transformación agrícola en Colombia, afirman que las UPA's que reciben el servicio de asistencia técnica son más productivas respecto a los que no perciben este servicio.

\section{Conclusiones}

Este artículo tuvo como objetivo caracterizar el estado actual de la asistencia técnica entre productores agrícolas de departamento de Bolívar, así como su incidencia en el rendimiento final de sus cultivos. Para ello, se acudió a las estadísticas del III Censo Nacional Agropecuario del año 2014, así como a la base de datos del Sistema de Información Agrícola -AGRONET- del Ministerio de Agricultura y Desarrollo Rural para el periodo entre el año 2010 - 2018. Los resultados encontrados de la caracterización situación actual del sector agrícola, en términos de asistencia técnica y el análisis del promedio total del rendimiento de las UPA's que reciben y no el servicio de asistencia técnica, permiten concluir que no existe mayor relevancia de la incidencia del servicio de asistencia técnica respecto al desempeño agrícola, medida en las toneladas producidas por hectárea. Lo anterior se pudo constatar al observar la mínima incidencia de cada una de las tipologías de asistencia técnica en el desempeño de UPA's.

Cabe aclarar que el servicio de asistencia técnica sigue siendo considerado por los autores como un factor importante en la tecnificación del campo colombiano, en aras de mejorar la competitividad a nivel internacional, para que se vea reflejado en una mejor calidad de vida de la población rural dedicada a la agricultura. Por ende, es importante seguir analizando sobre la calidad y la pertinencia de la asistencia técnica brindada a los productores agrícolas bolivarenses, así como otros factores que inciden en el desempeño agrícola, tales como la maquinaria, los efectos del cambio climático, la situación sociopolítica del país y el comportamiento de los jóvenes frente al interés de desarrollar actividades agrícolas como fuente de ingresos principal.

\section{Referencias}

\section{Alfaro, J. M. (2013). Desarrollo rural y ciudadanía social, territorios, instituciones y actores locales. 274.}

Berton, G. (2009). Apreciaciones conceptuales del término "Desarrollo." Huellas, 13(13), 192-203. http://search.ebscohost.com/login.aspx? direct $=$ true $\& d b=$ fua $\& A N=49846309 \& 1$ ang=es\&site $=$ ehost-live

Carro, R., \& González, D. (2012). Administración de Operaciones: Productividad y Competitividad.

Castaño Giraldo, N. E., \& Cardona Gómez, M. A. (2014). Factores determinantes en la inestabilidad del sector agrícola colombiano Determining factors in Colombian agricultural sector instability. En Contexto, 91-107.

Cepal, FAO, \& IICA. (2017). Perspectiva de la agricultura y del desarrollo rural en las americas: una mirada hacia América y el caribe. In Journal of Chemical Information and Modeling (Vol. 53, Issue https://doi.org/10.1017/CBO978110741 
Estado actual de la asistencia técnica y su incidencia en el desempeño agrícola en el departamento de bolívar

5324.004

Corredor Ríos, A. (2017). Empleo y productividad agropecuaria en Colombia. 151-186. https://doi.org/10.18356/784024d8-es

Cuevas Reyes, V., Baca del Moral, J., Cervantes Escoto, F., \& Aguilar Ávila, J. (2018). Asistencia técnica en el sector agropecuario en México: análisis del VIII censo agropecuario y forestal. Revista Mexicana de Ciencias Agrícolas, 3(5), 943-957. https://doi.org/10.29312/remexca.v3i5.1 386

DANE. (2015). Caracterización de los productores residentes en el área rural dispersa censada (p. 54). Departamento Administrativo Nacional de Estadísticas. https://doi.org/10.1007/s13398-0140173-7.2

DANE. (2016). III. Censo nacional agropecuario. Tomo 2, Resultados.

Gobernación de Bolívar. (2010). Plan regional de competitividad Cartagena y Bolivar.

Herrera Seba, G. A., \& Cumplido Hernandez, V. R. (2015). Implicaciones de la palma de aceite en la estructura productiva agrícola y la seguridad alimentaria del municipio de María La Baja - Bolívar. Universidad Tecnológica de Bolívar.

ICA. (2016). Resolucion No. 020009 del 07 Abril 2016 del Instituto Colombiano Agropecuario (pp. 1-29).

JIA, \& IICA. (2015). Una productividad competitiva, incluyente y sustentable: oportunidad para el continente americano (Vol. 370, Issue 15).

Landini, F. (2016). Problemas de la extensión rural en América Latina. Perfiles Latinoamericanos, 24(47), 47-68. https://doi.org/10.18504/pl2447-0052016

Leibovich, J., Perfettí, J. J., Botello, S., \& Vásquez, H. (2010). El proceso de transformación agricola en Colombia: Un análisis microeconómico. RegionES, $5,5-53$.

Lozano, I., \& Restrepo, J. C. (2015). El papel de la infraestructura rural en el desarrollo agrícola en Colombia (No. 904; Borradores de Economía).

Maza Avila, F. J. (2016). Diseño de políticas públicas para la transformación de las prácticas agrícolas de la región del Canal del Dique y Zona Costera Colombia-. Universidad de Cádiz.

Maza Ávila, F. J., Batista Ochoa, I. E., \& Rodriguez Arias, C. A. (2018). La incidencia de la asistencia técnica en el desempeño de las unidades productoras agropecuarias en la región caribe. The British Journal of Psychiatry, 111(479), 1009-1010. https://doi.org/10.1192/bjp.111.479.100 9-a

Ministerio de Agricultura y Desarrollo Rural. (1991). Decreto 2379 de 1991. 1991 (octubre 21), 15.

Ministerio de Agricultura y Desarrollo Rural. (2000). Ley 607. 2000(44), 11.

OCDE. (2014). Revisión de la OCDE de las politicas agrícolas: Colombia 2015 Evaluación y recomendaciones de 
política.

Perfetti, J. J., Balcázar, Á., Hernández, A., \& Leibovich, J. (2013). Políticas para el desarrollo de la agricultura en Colombia (Fedesarrollo, Sociedad de Agricultores deColombia (SAC), Incoder, Finagro, \& Banco Agrario (eds.); Primera). Fedesarrollo.

Presidencia de la República, \& FARC-EP. (2016). Acuerdo final para la terminación del conflicto y la construcción de una paz estable y duraderas Se Produjo Un. Acuerdo Final Para La Terminación Del Conflicto Y La Construcción De Una Paz Estable Y Duradera, 1-310.

Procasur, Ministerio de Agricultura y Desarrollo Rural, \& Fordfundation. (2016). Sistema de asistencia técnica y capacitación rural en Colombia y perspectivas para la integración de talentos rurales (p. 70). Ministerio de Agricultura y Desarrollo rural. https://doi.org/10.1192/bjp.111.479.100 9-a

Valdés Alonso, A., Foster, W., \& Rivera, R. (2008). Evolución del ingreso agrícola real en América Latina, 1990-2005: evidencia en base a cuentas nacionales y encuestas de hogares. Revista Española de Estudios Agrosociales y Pesqueros, 218, 71-98.

Villanueva-Mejía, D. F. (2018). Estudios sobre la Bioeconomía como fuente de nuevas industrias basadas en el capital natural de Colombia. Estudios Sobre La Bioeconomía Como Fuente de Nuevas Industrias Basadas En El Capital Natural de Colombia, 1-49. 